\title{
The extent of colorectal resection and short-term outcomes in patients with ulcerative colitis
}

\author{
Mohamed A. Abd El Aziz ${ }^{1}$. William R. G. Perry ${ }^{1} \cdot$ Fabian Grass $^{1} \cdot$ Amit Merchea $^{2} \cdot$ Laura E. Raffals $^{3} \cdot$ Kellie L. Mathis ${ }^{1}$. \\ Kevin T. Behm ${ }^{1}$
}

Received: 11 February 2021 / Accepted: 22 March 2021 / Published online: 30 March 2021

(C) Italian Society of Surgery (SIC) 2021

\begin{abstract}
Objective There is limited literature on the impact of the extent of resection on short-term outcomes in patients with ulcerative colitis (UC) in an elective setting. The aim of this study was to better understand the impact of approach and extent of resection on short-term outcomes for patients undergoing total proctocolectomy (TPC) and subtotal colectomy (STC) for UC. Methods Patients with UC who underwent elective TPC or STC were captured from the ACS-NSQIP® 2011-2018 database and divided into four cohorts: Open TPC (O-TPC), Laparoscopic TPC (L-STC), Open STC (O-STC), and Laparoscopic STC (L-STC). Baseline and perioperative variables were compared between the four groups alongside 30-day mortality and 30-day complication rates.

Results Of 3387 patients, 368 (10.9\%) underwent O-STC, 406 (12\%) underwent O-TPC, 1958 (58\%) underwent L-STC, and $655(19 \%)$ underwent L-TPC. Overall rate of prolonged length of stay (LOS) was $27 \%$ and $9 \%$ needed a blood transfusion. There was no difference in the risk of complications between open TPC and open STC. Those who had open surgery had a higher risk of complications and prolonged LOS. Patients who had L-TPC had prolonged LOS compared to patients who had L-STC, but less compared to those who had O-STC.

Conclusion Elective surgery for UC is associated with high rates of prolonged LOS and blood transfusion despite MIS approaches. Short-term outcomes and LOS are more impacted by the operative approach than the extent of resection. Despite this laparoscopic TPC has higher rates of prolonged LOS when compared to laparoscopic STC.
\end{abstract}

Keywords Ulcerative colitis $\cdot$ Total proctocolectomy $\cdot$ Subtotal colectomy $\cdot$ Laparoscopic surgery $\cdot$ Open surgery

\section{Introduction}

Despite advances in the medical management of Ulcerative Colitis (UC), the incidence rate of colectomies is still estimated to be approximately 30 per 1000 patients per year [1]. Elective surgery is offered for patients who have a refractory disease for medical management, dysplasia, or invasive malignancy.

Kevin T. Behm

behm.kevin@mayo.edu

1 Division of Colon and Rectal Surgery, Department of Surgery, Mayo Clinic, Rochester, MN, USA

2 Division of Colon and Rectal Surgery, Department of Surgery, Mayo Clinic, Jacksonville, FL, USA

3 Division of Gastroenterology and Hepatology, Mayo Clinic, Rochester, MN, USA
The extent of the operation is dependent on patients' clinical condition as well as patients' preference for restorative versus non-restorative surgery. The index operation is most commonly a non-restorative subtotal colectomy (STC) with end ileostomy or total proctocolectomy (TPC) with end ileostomy [2]. However, most will seek eventual restoration and undergo a completion proctectomy with the formation of an ileal pouch-anal anastomosis (IPAA). [2].

Minimally invasive techniques have dramatically improved short-term surgical outcomes in patients with UC [3]. Intuitively, operative approach was thought to be the most valuable factor that affects the postoperative outcome regardless of the extent of resection. Limited literature exists on the impact of the interaction between operative approach and extent of resection on short-term outcomes and length of stay (LOS) for patients undergoing surgery UC in an elective setting. This study aimed to better understand the impact of 
surgical approach and extent of resection on these outcomes for patients undergoing TPC and STC for UC.

\section{Materials and methods}

The American College of Surgeons National Surgical Quality Improvement Program database (ACS-NSQIP®), was interrogated. ACS-NSQIP® is a validated, risk-adjusted, and outcome-based national program developed for quality improvement purposes. Data abstraction from all participant sites is conducted by trained abstractors. A random sample of $20 \%$ of patients from each contributing institution is included in the final dataset $[4,5]$.

After merging the main ACS-NSQIP® Participant User Files (PUF) from 2011 to 2018, we identified patients who had an open or laparoscopic colectomy (total or subtotal) using the Current Procedure Terminology (CPT) codes: 44,150 and 44,210 for subtotal colectomy and 44,155 and 44,212 for total colectomy. Patients who had IPAA were excluded. We then used the International Classification of Disease (ICD9: 556.xx and ICD 10: K51.xx) codes to identify patients who had surgery for UC. This study only considered those who underwent elective non-urgent surgery. The ACS-NSQIP defines elective surgery as surgery performed for a patient who was well enough to present from home only for a prescheduled surgery. Patients who had a concurrent operation under the responsibility of another surgeon were excluded from this study (Fig. 1).

Patients were divided into four groups according to the extent of resection and the surgical approach: Open Total
Proctocolectomy (O-TPC), Laparoscopic Total Proctocolectomy (L-TPC), Open Subtotal Colectomy (O-STC), and Laparoscopic Subtotal Colectomy (L-STC). Baseline demographic, anthropometric, and perioperative risk factors were compared between the three groups. Medical and surgical 30-day complications as defined by ACS NSQIP ${ }^{\circledR}$ were subsequently assessed alongside the length of index hospital stay (LOS). Prolonged LOS was defined as $>6$ days, the third quartile of the LOS for the whole cohort (Fig. 2).

\section{Statistical analysis}

Data were summarized by the median (interquartile range) for continuous variables or frequencies and percentages for categorical variables. Multigroup comparisons were performed using the Independent Samples Kruskal-Wallis Test for continuous variables and the Chi-squared test for categorical variables. Binary logistic regression analysis was used to adjust for the baseline confounders. The analysis was conducted using the Statistical Package for Social Sciences (SPSS, Version 25; SPSS, Inc., Armonk, NY, USA). A p-value of less than 0.05 was considered significant. All tests were two-sided.

\section{Results}

A total of 3387 patients were identified. Patient demographics and perioperative characteristics are presented in Table 1. Some 368 (10.9\%) patients underwent O-STC, 406 (12\%) underwent O-TPC, 1958 (58\%) underwent L-STC, and 655

Fig. 1 Study flow diagram

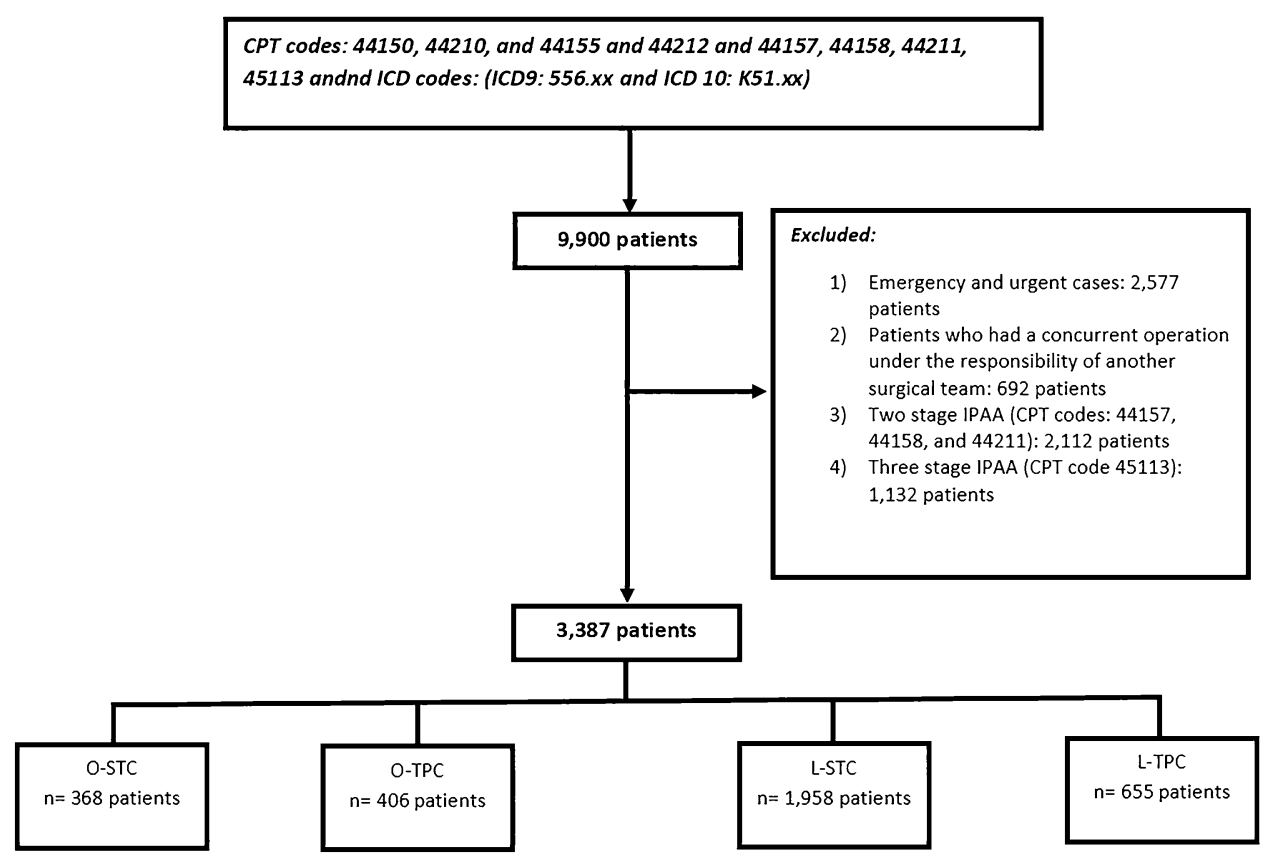


Fig. 2 Multivariable logistic regression analysis. SSI: surgical site infection, BL: blood, LOS: length of stay, UTI: urinary tract infection, STC: subtotal colectomy, TPC: total proctocolectomy, L: laparoscopic, O: open. *Adjusted for age, BMI, DM, Dyspnea, COPD, HTN, chronic steroid use, $>10 \%$ loss of body weight, bleeding disorder, ASA $\geq 3$, and systemic sepsis, operation time

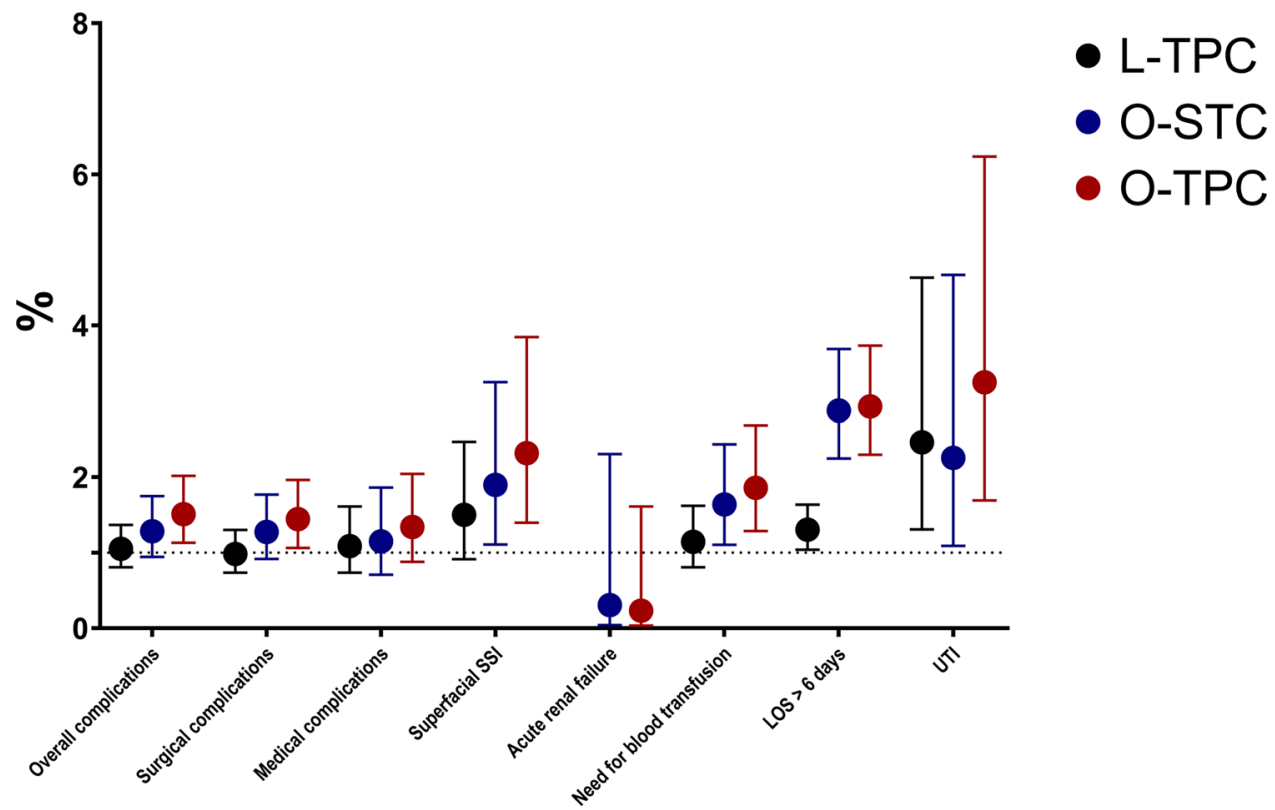

Table 1 Baseline demographic, anthropometric, and perioperative characteristics

\begin{tabular}{|c|c|c|c|c|c|}
\hline & $\begin{array}{l}\text { O-STC } \\
N=368\end{array}$ & $\begin{array}{l}\text { O-TPC } \\
N=406\end{array}$ & $\begin{array}{l}\text { L-STC } \\
N=1958\end{array}$ & $\begin{array}{l}\text { L-TPC } \\
N=655\end{array}$ & $\begin{array}{l}\text { Total } \\
N=3387\end{array}$ \\
\hline Age $>80$ & $7(1.9 \%)$ & $11(2.7 \%)$ & $13(0.7 \%)$ & $11(1.7 \%)$ & $42(1.2 \%)$ \\
\hline Age; years (Median; IQR) & $47(32-58)$ & $59(45-68)$ & $38(28-53)$ & $57(42-68)$ & $45(31-60)$ \\
\hline Male & $225(61.1 \%)$ & $245(60.3 \%)$ & $1148(58.6 \%)$ & $351(53.6 \%)$ & $1969(58.1 \%)$ \\
\hline BMI: median (IQR), $30 \mathrm{~kg} / \mathrm{m}^{2}$ & $28(24-33)$ & $27(24-32)$ & $25(22-30)$ & $26(23-30)$ & $26(22-31)$ \\
\hline $\mathrm{BMI}>30 \mathrm{~kg} / \mathrm{m}^{2}$ & $141(39 \%)$ & $141(34.9 \%)$ & $473(24.3 \%)$ & $162(24.8 \%)$ & $917(27.2 \%)$ \\
\hline DM & $38(10.3 \%)$ & $52(12.8 \%)$ & $127(6.5 \%)$ & $65(9.9 \%)$ & $282(8.3 \%)$ \\
\hline Current smoker & $26(7.1 \%)$ & $27(6.7 \%)$ & $120(6.1 \%)$ & $36(5.5 \%)$ & $209(6.2 \%)$ \\
\hline Dyspnea & $21(5.7 \%)$ & $22(5.4 \%)$ & $61(3.1 \%)$ & $32(4.9 \%)$ & $136(4.0 \%)$ \\
\hline Functional status; Independent & $364(98.9 \%)$ & $402(99.0 \%)$ & $1944(99.3 \%)$ & $654(99.8 \%)$ & $3364(99.3 \%)$ \\
\hline COPD & $4(1.1 \%)$ & $11(2.7 \%)$ & $14(0.7 \%)$ & $15(2.3 \%)$ & $44(1.3 \%)$ \\
\hline Ascites & $2(0.5 \%)$ & 0 & $2(0.1 \%)$ & $1(0.2 \%)$ & $5(0.1 \%)$ \\
\hline $\mathrm{CHF}$ & $1(0.3 \%)$ & $1(0.2 \%)$ & $2(0.1 \%)$ & $3(0.5 \%)$ & $7(0.2 \%)$ \\
\hline HTN requiring medication & $96(26.1 \%)$ & $159(39.2 \%)$ & $316(16.1 \%)$ & $203(31.0 \%)$ & $774(22.9 \%)$ \\
\hline Open wound/ wound infection & $4(1.1 \%)$ & $6(1.5 \%)$ & $14(0.7 \%)$ & $2(0.3 \%)$ & $26(0.8 \%)$ \\
\hline Steroid use for chronic condition & $248(67.4 \%)$ & $213(52.5 \%)$ & $1459(74.5 \%)$ & $362(55.3 \%)$ & $2282(67.4 \%)$ \\
\hline$>10 \%$ loss of body weight in last 6 months & $32(8.7 \%)$ & $25(6.2 \%)$ & $201(10.3 \%)$ & $42(6.4 \%)$ & $300(8.9 \%)$ \\
\hline Bleeding disorder & $23(6.3 \%)$ & $8(2.0 \%)$ & $41(2.1 \%)$ & $24(3.7 \%)$ & $96(2.8 \%)$ \\
\hline $\begin{array}{l}\text { Transfusion of } \geq 1 \text { unite pRBCs within } 72 \mathrm{~h} \\
\text { before surgery }\end{array}$ & $10(2.7 \%)$ & $4(1.0 \%)$ & $34(1.7 \%)$ & $5(0.8 \%)$ & $53(1.6 \%)$ \\
\hline Systemic sepsis & $8(2.2 \%)$ & $6(1.5 \%)$ & $77(3.9 \%)$ & $20(3.1 \%)$ & $111(3.3 \%)$ \\
\hline ASA Class $\geq 3$ & $200(54.3 \%)$ & $229(56.4 \%)$ & $790(40.3 \%)$ & $311(47.5 \%)$ & $1530(45.2 \%)$ \\
\hline Operation time; minutes (Median; IQR) & $175(127-235)$ & $242(182-320)$ & $196(156-246)$ & $280(223-353)$ & $211(162-278)$ \\
\hline
\end{tabular}

$S T C$ subtotal colectomy, $T P C$ total proctocolectomy, $L$ Laparoscopic, $O$ open $D M$ diabetes mellitus, $C O P D$ chronic obstructive pulmonary disease, $C H F$ cardiac incident before the operation, HTN hypertension, pRBCs packed Red Blood Cells, hrs hours, ASA American Society of Anesthesiologists

(19.3\%) underwent L-TPC. The overall median operative time was 211 (IQR 162-278) minutes. A total of 297 (8.8\%) needed blood transfusion and $917(27.1 \%)$ required admission for $>6$ days. 


\section{Extent of the surgery}

Patients who had TPC were more likely to have higher preoperative comorbidities and longer median operation time (O-TPC $242 \mathrm{~min}$ (IQR 182-320) vs. O-STC 175 (127-235); L-TPC 280 (223-353) vs. L-STC (196 (156-246)). They also had a higher risk of superficial SSI, need for blood transfusion, and need for prolonged LOS (Table 2). After adjusting for the baseline confounders, there was no significant difference in the risk of complications according to the extent of surgery for patients who had open surgery (Fig. 1). However, patients who had L-TPC had a higher risk of urinary tract infection (UTI) and prolonged LOS compared to patients who had L-STC.

\section{Operative approach}

When the extent of the surgery was the same, patients who had open surgery had higher rates of preoperative comorbidities such as diabetes mellitus, chronic obstructive pulmonary disease, and an ASA $\geq 3$ (Table 1). They were also more likely to have a shorter operation time (O-STC $175 \mathrm{~min}$ (127-235) vs. L-STC 196 (156-246); O-TPC 242 (182-320) vs. L-TPC 280 (223-353)) and a higher risk of postoperative complications including superficial SSI, acute renal failure, need for blood transfusion, and prolonged LOS (Table 2). There was no significant difference between the four groups regarding 30-day mortality rate, organ space infection, unplanned reoperation, cardiovascular or pulmonary complications. After adjusting for the baseline confounders, open surgery was an independent risk factor for the need for prolonged LOS compared to the laparoscopic approach (Fig. 1).

Table 2 Post-operative complications

\begin{tabular}{|c|c|c|c|c|c|c|}
\hline & $\begin{array}{l}\text { O-STC } \\
N=368\end{array}$ & $\begin{array}{l}\text { O-TPC } \\
N=406\end{array}$ & $\begin{array}{l}\text { L-STC } \\
N=1958\end{array}$ & $\begin{array}{l}\text { L-TPC } \\
N=655\end{array}$ & $\begin{array}{l}\text { Total } \\
N=3387\end{array}$ & $P$ value \\
\hline Overall complications & $99(26.9 \%)$ & $140(34.5 \%)$ & $401(20.5 \%)$ & $181(27.6 \%)$ & $821(24.2 \%)$ & $<0.0001$ \\
\hline Overall surgical & $83(22.6 \%)$ & $116(28.6 \%)$ & $330(16.9 \%)$ & $144(22.0 \%)$ & $673(19.9 \%)$ & $<0.0001$ \\
\hline Overall medical & $33(9.0 \%)$ & $49(12.1 \%)$ & $133(6.8 \%)$ & $63(9.6 \%)$ & $278(8.2 \%)$ & 0.002 \\
\hline Superficial Incisional SSI & $20(5.4 \%)$ & $29(7.1 \%)$ & $55(2.8 \%)$ & $31(4.7 \%)$ & $135(4.0 \%)$ & $<0.0001$ \\
\hline Deep incisional SSI & $8(2.2 \%)$ & $6(1.5 \%)$ & $20(1.0 \%)$ & $6(0.9 \%)$ & $40(1.2 \%)$ & 0.237 \\
\hline Organ/Space SSI & $17(4.6 \%)$ & $26(6.4 \%)$ & $85(4.3 \%)$ & $39(6.0 \%)$ & $167(4.9 \%)$ & 0.181 \\
\hline Wound disruption & $7(1.9 \%)$ & $5(1.2 \%)$ & $17(0.9 \%)$ & $6(0.9 \%)$ & $35(1.0 \%)$ & 0.323 \\
\hline Pneumonia & $7(1.9 \%)$ & $6(1.5 \%)$ & $20(1.0 \%)$ & $9(1.4 \%)$ & $42(1.2 \%)$ & 0.5 \\
\hline Unplanned intubation & $2(0.5 \%)$ & $4(1.0 \%)$ & $12(0.6 \%)$ & $7(1.1 \%)$ & $25(0.7 \%)$ & 0.588 \\
\hline Pulmonary embolism & $2(0.5 \%)$ & $4(1.0 \%)$ & $9(0.5 \%)$ & $2(0.3 \%)$ & $17(0.5 \%)$ & 0.477 \\
\hline On mechanical ventilator $>48 \mathrm{~h}$ & $3(0.8 \%)$ & $4(1.0 \%)$ & $10(0.5 \%)$ & $4(0.6 \%)$ & $21(0.6 \%)$ & 0.685 \\
\hline Progressive renal insufficiency & $5(1.4 \%)$ & $4(1.0 \%)$ & $8(0.4 \%)$ & $4(0.6 \%)$ & $21(0.6 \%)$ & 0.135 \\
\hline Acute renal failure & $2(0.5 \%)$ & $3(0.7 \%)$ & $2(0.1 \%)$ & 0 & $7(0.2 \%)$ & 0.019 \\
\hline UTI & $11(3.0 \%)$ & $19(4.7 \%)$ & $26(1.3 \%)$ & $23(3.5 \%)$ & $79(2.3 \%)$ & $<0.0001$ \\
\hline Stroke & 0 & $2(0.5 \%)$ & $1(0.1 \%)$ & $2(0.3 \%)$ & $5(0.1 \%)$ & 0.104 \\
\hline Cardiac arrest requiring CPR & 0 & $2(0.5 \%)$ & $2(0.1 \%)$ & $3(0.5 \%)$ & $7(0.2 \%)$ & 0.144 \\
\hline Myocardial infarction & $1(0.3 \%)$ & $2(0.5 \%)$ & $3(0.2 \%)$ & $1(0.2 \%)$ & $7(0.2 \%)$ & 0.562 \\
\hline Need for blood transfusion & $39(10.6 \%)$ & $54(13.3 \%)$ & $140(7.2 \%)$ & $64(9.8 \%)$ & $297(8.8 \%)$ & $<0.0001$ \\
\hline Deep venous thrombosis & $10(2.7 \%)$ & $13(3.2 \%)$ & $67(3.4 \%)$ & $27(4.1 \%)$ & $117(3.5 \%)$ & 0.669 \\
\hline Sepsis & $20(5.4 \%)$ & $21(5.2 \%)$ & $68(3.5 \%)$ & $21(3.2 \%)$ & $130(3.8 \%)$ & 0.116 \\
\hline Septic Shock & $5(1.4 \%)$ & $7(1.7 \%)$ & $17(0.9 \%)$ & $4(0.6 \%)$ & $33(1.0 \%)$ & 0.256 \\
\hline Unplanned reoperation & $17(4.6 \%)$ & $18(4.4 \%)$ & $107(5.5 \%)$ & $27(4.1 \%)$ & $169(5.0 \%)$ & 0.504 \\
\hline $\operatorname{LOS}>6$ days & $147(39.9 \%)$ & $196(48.4 \%)$ & $369(18.8 \%)$ & $205(31.4 \%)$ & $917(27.1 \%)$ & $<0.0001$ \\
\hline 30 day mortality & $1(0.3 \%)$ & $1(0.2 \%)$ & $2(0.1 \%)$ & $3(0.5 \%)$ & $7(0.2 \%)$ & 0.369 \\
\hline
\end{tabular}

STC: subtotal colectomy, TPC: total proctocolectomy, L: Laparoscopic, O: open, SSI: surgical site infection, UTI: Urinary tract infection, CPR: Cardiopulmonary resuscitation, LOS: length of stay

Overall surgical including: any SSI, wound disruption, need for blood transfusion, sepsis, septic shock, and unplanned reoperation

Overall medical including pneumonia, unplanned intubation, on mechanical ventilation for $>48 \mathrm{~h}$, progressive renal insufficiency, acute renal failure, urinary tract infection, myocardial infarction, cardiac arrest requiring CPR, stroke, and urinary tract infection

Overall complications includes surgical or medical complications 


\section{Interaction between the operative approach and extent of surgery}

\section{O-STC versus L-TPC}

Patients who had O-STC were more likely to have higher preoperative co-morbidities (Table 1). They had a shorter operation time, a higher risk of superficial SSI, acute renal failure, and an associated prolonged LOS. There was no significant difference between the groups regarding the 30-day mortality rate, organ space infection, systemic sepsis, respiratory or cardiac complications. Patients who had L-TPC were at a higher risk for the need for blood transfusion (Table 2). After adjusting for the baseline confounders, patients who had O-STC were more likely to have a prolonged LOS but there was no significant difference between the two groups regarding SSI, need for blood transfusion, and UTI (Fig. 1).

\section{O-TPC versus L-STC}

Again, patients who had O-TPC were more likely to have more preoperative comorbidities. They also had longer operation time and a higher risk of superficial SSI, acute renal failure, need for blood transfusion, and prolonged LOS (Table 2). After adjusting for the baseline confounders, patients who had O-TPC had a higher risk of superficial SSI, need for blood transfusion, and prolonged LOS (Fig. 1).

\section{Discussion}

This nationally representative sample compared both the approach and extent of elective surgery for elective patients with ulcerative colitis. There are several surgical strategies available to a patient and his/her surgeon depending on patient characteristics, disease status, and desire for restorative surgery. In this study, we focused solely on the primary procedure regardless of subsequent planned operations.

Fifty-eight percent of patients underwent a L-STC, reflective of a trend towards MIS, that allows for future restorative surgery (IPAA or IRA). This is consistent with other studies [6]. Of note, nearly one-third of patients had a length of stay of more than six days despite elective surgery and the nationwide drive towards enhanced recovery pathways [7]. Furthermore, nearly 1 in 10 required a blood transfusion. These findings highlight the high complication rates in patients with ulcerative colitis even after elective surgery which is consistent with what has been published before [8].

There was no significant difference in the risk of complications between open STC and open TPC suggesting most of the morbidity was associated with the open nature of the procedure. This notion is further supported by the fact that patients who had L-TPC had a higher adjusted risk of UTI and prolonged LOS than those who had L-STC. Overall, open surgery was an independent risk factor for the need for blood transfusion and prolonged LOS compared to the laparoscopic approach.

Interestingly, O-STC was associated with a higher risk of LOS compared to both L-STC and L-TPC. Moreover, L-TPC was associated with a higher risk of LOS compared to L-STC. Similar results were noted from other tertiary care centers $[9,10]$. The Oxford group's overall mortality rate was also comparable to our results $<0.1 \%$ [9].

From a surgical perspective, there is scant data about comparing STC and TPC in a completely elective setting, as STC has traditionally been reserved for patients who have a higher risk for postoperative complications [2]. Indeed in this study, L-STC carried the least short-term morbidity and LOS. Such an approach is particularly useful in the setting of a female patient in their reproductive years [11-13].

This study has two key findings. First, it highlights the role of minimally invasive surgery where appropriate. Regardless of the extent, laparoscopic patients had a lower risk of blood transfusion and prolonged length of stay. This is consistent with other MIS literature [14-16]. Second, elective surgery for ulcerative colitis is associated with prolonged LOS even with MIS. This is important for surgeons to understand when planning for surgery and informing patients of their expected hospital stay. Further, this supports a greater focus on prehabilitation to minimize LOS.

The study has several limitations. Although we have adjusted for the available baseline confounders, we could not adjust for some unmeasured confounders such as surgeons' experience and the MIS case numbers in each center. Further, the STC groups also captured what is likely a small number of patients who had a concurrent ileorectal anastomosis as CPT codes do not allow for differentiation. However, given this involves an anastomosis, exclusion of this subset would likely only improve outcomes of these groups. In our study, patients who had STC (open or MIS) had a higher proportion of chronic immunosuppressives use highlighting the severity of the disease. For patients who had TPC, the rates of chronic immunosuppressives use were lower than STC cohorts (Table 1). Immunosuppressives use was included in the regression model to account for the impact on LOS and postoperative operative morbidity. Unfortunately, specifics about the class of immunosuppressive medications are not recorded in the ACS-NSQIP limiting our ability to further explore this important relationship. Finally, further planned restorative or non-restorative procedures, functional outcomes, long-term complications, and quality of life could not be assessed. It does, however, provide a good macroscopic understanding of surgical practice for ulcerative colitis in the elective setting and helps inform future studies. 


\section{Conclusion}

Elective surgery for UC is associated with high rates of prolonged LOS and blood transfusion despite MIS approaches. Short-term outcomes and LOS are more impacted by the operative approach than the extent of resection. Despite this finding laparoscopic TPC has higher rates of prolonged LOS when compared to laparoscopic STC.

\section{Declarations}

Conflict of interest The authors declare that they have no conflict of interest.

Research involving human participants and/or animals This article does not contain any experimental studies with human participants or animals performed by any of the authors.

Informed consent For this type of study formal consent is not required.

\section{References}

1. Abou Khalil M, Boutros M, Nedjar H, Morin N, Ghitulescu G, Vasilevsky CA, Gordon P, Rahme E (2018) Incidence rates and predictors of colectomy for ulcerative colitis in the era of biologics: results from a Provincial database. J Gastrointest Surg 22(1):124-132. https://doi.org/10.1007/s11605-017-3530-y

2. Murphy B, Kavanagh DO, Winter DC (2020) Modern surgery for ulcerative colitis. Updates Surg. https://doi.org/10.1007/ s13304-020-00719-4

3. Causey MW, Stoddard D, Johnson EK, Maykel JA, Martin MJ, Rivadeneira D, Steele SR (2013) Laparoscopy impacts outcomes favorably following colectomy for ulcerative colitis: a critical analysis of the ACS-NSQIP database. Surg Endosc 27(2):603609. https://doi.org/10.1007/s00464-012-2498-7

4. Ingraham AM, Richards KE, Hall BL, Ko CY (2010) Quality improvement in surgery: the American College of surgeons national surgical quality improvement program approach. Adv Surg 44:251-267. https://doi.org/10.1016/j.yasu.2010.05.003

5. Shiloach M, Frencher SK Jr, Steeger JE, Rowell KS, Bartzokis K, Tomeh MG, Richards KE, Ko CY, Hall BL (2010) Toward robust information: data quality and inter-rater reliability in the American College of Surgeons National Surgical Quality Improvement Program. J Am Coll Surg 210(1):6-16. https://doi.org/10.1016/j. jamcollsurg.2009.09.031

6. Loftus EV Jr, Delgado DJ, Friedman HS, Sandborn WJ (2008) Colectomy and the incidence of postsurgical complications among ulcerative colitis patients with private health insurance in the United States. Am J Gastroenterol 103(7):1737-1745. https:// doi.org/10.1111/j.1572-0241.2008.01867.x

7. Pędziwiatr M, Mavrikis J, Witowski J, Adamos A, Major P, Nowakowski M, Budzyński A (2018) Current status of enhanced recovery after surgery (ERAS) protocol in gastrointestinal surgery. Med Oncol (Northwood, London, England) 35(6):95. https://doi.org/ 10.1007/s12032-018-1153-0

8. Baker DM, Folan AM, Lee MJ, Jones GL, Brown SR, Lobo AJ (2020) A systematic review and meta-analysis of outcomes after elective surgery for ulcerative colitis. Colorectal Dis. https://doi. org/10.1111/codi.15301

9. Buchs NC, Bloemendaal ALA, Wood CPJ, Travis S, Mortensen NJ, Guy RJ, George BD (2017) Subtotal colectomy for ulcerative colitis: lessons learned from a tertiary centre. Colorectal Dis 19(5):O153-O161. https://doi.org/10.1111/codi.13658

10. Chung TP, Fleshman JW, Birnbaum EH, Hunt SR, Dietz DW, Read TE, Mutch MG (2009) Laparoscopic vs. open total abdominal colectomy for severe colitis: impact on recovery and subsequent completion restorative proctectomy. Dis Colon Rectum 52 (1):4-10. doi:https://doi.org/10.1007/DCR.0b013e3181975701

11. Waljee A, Waljee J, Morris AM, Higgins PD (2006) Threefold increased risk of infertility: a meta-analysis of infertility after ileal pouch anal anastomosis in ulcerative colitis. Gut 55(11):15751580. https://doi.org/10.1136/gut.2005.090316

12. Gorgun E, Remzi FH, Goldberg JM, Thornton J, Bast J, Hull TL, Loparo B, Fazio VW (2004) Fertility is reduced after restorative proctocolectomy with ileal pouch anal anastomosis: a study of 300 patients. Surgery 136(4):795-803. https://doi.org/10.1016/j.surg. 2004.06.018

13. Lepisto A, Sarna S, Tiitinen A, Jarvinen HJ (2007) Female fertility and childbirth after ileal pouch-anal anastomosis for ulcerative colitis. Br J Surg 94(4):478-482. https://doi.org/10.1002/bjs.5509

14. Bartels SA, Gardenbroek TJ, Ubbink DT, Buskens CJ, Tanis PJ, Bemelman WA (2013) Systematic review and meta-analysis of laparoscopic versus open colectomy with end ileostomy for nontoxic colitis. Br J Surg 100(6):726-733. https://doi.org/10.1002/ bjs. 9061

15. Abd El Aziz MA, Grass F, Behm KT, Shawki S, D’Angelo A-L, Mathis KL, Larson DW (2020) Trends of complications and innovative techniques' utilization for colectomies in the United States. Updat Surg. https://doi.org/10.1007/s13304-020-00862-y

16. Abd El Aziz MA, Grass F, Perry W, Behm KT, Shawki SF, Larson DW, Mathis KL (2020) Colectomy for patients with super obesity: current practice and surgical morbidity in the United States. Surg Obesity Relat Dis. https://doi.org/10.1016/j.soard.2020.06.033

Publisher's Note Springer Nature remains neutral with regard to jurisdictional claims in published maps and institutional affiliations. 